\title{
Elucidating Angiogenesis: The Role of Basement Membrane Proteolysis and Endothelial Cell Motility and Proliferation
}

\section{Neil A. Goldenberg*}

* To whom correspondence should be addressed: Faculty of Medicine, McGill University, Montreal, QC, Canada H3G 1Y6

\section{INTRODUCTION}

The process of blood vessel neoformation from pre-existing microvasculature, known as angiogenesis, has in recent years become the focus of intense interest in diverse fields of basic scientific and clinical research. De novo blood vessel growth appears to play a vital role in early development, as well as in various physiological and pathological settings. Like vasculogenesis (the development of vascular structures from mesenchymal tissue), angiogenesis is a fundamental feature of embryonic development and organogenesis, as circulatory branching is essential in sustaining newly developing structures and tissues. In the adult, angiogenesis is physiologically significant in its association with inflammation, wound healing, and placental formation. The redness associated with the "wheal and flare" inflammatory reaction is caused by capillary dilation (1) and neogenesis (2) at the site of tissue damage. Similarly, in a healing wound, new blood vessels arise in the developing replacement tissues as well as in the granulation tissue near necrotic regions (3). In pregnancy, organogenesis occurs with the formation of the placenta. The developing placenta requires dense capillary perfusion, which is met by rapid angiogenesis.

Angiogenesis also plays a primary role in several diseases. In addition to tumor growth and metastasis, aberrant regulation of angiogenesis is implicated in rheumatoid arthritis, in which an autoimmune inflammatory reaction directs capillary neogenesis in the joints. These capillaries may invade and destroy cartilage $(4,5)$. Likewise, chronic inflammation is characterized by protracted angiogenesis, presumably due to continual induction of de novo vessel formation (2). In diabetic retinopathy, neovascular proliferation and macular edema lead to blindness (6). Finally, as de novo capillary production is thought to be the crucial event in tumor growth and metastasis (3), it is hoped that research into the cellular and molecular mechanisms underlying angiogenesis will yield effective cancer therapies through the inhibition and/or disruption of tumor vascularization.

Angiogenesis is both critical to, and significantly parallels, tumor metastasis. In metastasis, neoplastic cells detach from the primary tumor mass and degrade the basement membrane of an adjacent vessel prior to traveling through the vascular system. They may then extravasate at a subsequent capillary bed, invade and adhere to a secondary tissue site, and proliferate to form a new tumor mass. Angiogenesis shares some of these features, as revealed by the induction of angiogenesis by tumors implanted in rabbit corneas and chick 
chorioallantoic membranes, as well as by early investigations involving endothelial cell cultures (7). Based on observations in these studies, Judah Folkman, the pioneer of angiogenesis research, proposed the following chronology of events in the neovascularization process $(2,4)$ : (i) local basement membrane degradation of the parent vessel by endothelial cells (commonly an existing capillary or post-capillary venule), (ii) locomotion of endothelial cells away from the parent vessel in the direction of an angiogenic stimulus, (iii) elongation and alignment of migratory endothelial cells to form a capillary sprout, (iv) endothelial cell proliferation in the parent venule and in the capillary sprout, (v) lumen formation, (vi) anastomosis of two hollow sprouts to form a capillary loop, (vii) onset of blood flow, and (viii) production of, and pericyte incorporation into, the new basement membrane.

\section{PRINCIPAL METHODS OF INVESTIGATION}

Originally, the study of the angiogenic process was greatly limited by the lack of feasible, biologically relevant in vivo and in vitro models. However, the unveiling of the fundamental features of the neovascularization process has led to the refinement of various models and assays with which to study angiogenesis. These studies have primarily addressed three central elements of the angiogenic process: endothelial cell-mediated basement membrane degradation, endothelial cell motility, and endothelial cell proliferation,

In vitro studies of basement membrane degradation commonly employ endothelial cells cultured on a substrate designed to simulate the extracellular matrix. The substrates include gels composed of collagen, fibrin, or Matrigel, a laminin-rich gel recently developed from reconstituted basement membrane proteins (8). These in vitro models of angiogenesis involve endothelial cell attachment to the extracellular matrix, proteolytic invasion of the matrix in response to exogenous angiogenic inducers, endothelial cell alignment, and formation of capillary-like tubes. Tumor cell cultures have also been used as part of in vitro assays of angiogenesis (9).

These models have also been used, often with the administration of presumed angiogenic modulatory factors, to study endothelial cell migration and proliferation. In vivo research on cell migration and proliferation has utilized the rabbit cornea and chick embryo chorioallantoic membranes. In the first model, protein-polymer pellets implanted into the rabbit cornea serve as a sustained release system for angiogenic factors; the degree of neovascularization is subsequently quantified $(4,10)$. Alternatively, the degree to which angiogenesis inhibitors (also called "anti-angiogenic" or "angiostatic" substances) prevent the neovascularization normally induced by a tumor implant can be determined. The vascular chorioallantoic membrane of the chick embryo provides a more rapid assay based on a similar methodology $(4,11)$. In this system, the embryo is incubated without its shells for several days, after which time tumors or sustained-release discs are implanted into the chorioallantoic membrane. An additional in vivo system has recently been developed in which Matrigel, supplemented with heparin and fibroblast growth factor (FGF), is injected subcutaneously (12). The basement membrane proteins reconstitute a gel that is intensely vascularized within a few days, upon which the effects of angiogenic modulators can be assessed. Lastly, several tumor models can serve as in vivo assays of angiogenesis. These include tumors grown through one of several mechanisms: direct tumor transplantation into animals (4), subcutaneous injection of human tumor cell suspensions into athymic mice (13), and expression of oncogenes in transgenic mice (14).

\section{ROLE OF BASEMENT MEMBRANE PROTEOLYSIS}

Endothelial cells line the interior of blood vessels and are ensheathed by a basement membrane, a specialized form of the extracellular matrix comprised of collagen, glycoproteins such as fibronectin and laminin, and heparan sulfate proteoglycans. A crucial early step in the neovascularization process is the dissolution of the basement membrane at the site of endothelial outgrowth from the parent vessel, which facilitates cell migration and stromal invasion. The phenomenon of cell invasiveness is not unique to angiogenesis, but is 
also an essential component of both tumor metastasis and embryonic morphogenesis. Examples of the latter include primary and secondary mesenchymal cell invasion of the blastocoel in sea urchin gastrulae, the invasion of somites by migrating neural crest cells in vertebrate embryos, and fibroblast invasion of the developing cornea (15).

While the migration of single cells could presumably occur without matrix degradation, endothelial cell invasion, which involves penetration by multicellular tubes, cannot. Endothelial cells are normally noninvasive in vitro, forming a monolayer on a collagen matrix. In this respect, they differ markedly from other mesenchyme-derived cells, such as fibroblasts, leukocytes, and smooth muscle cells, that actively move through collagen substrate $(16,17)$. Investigations of the cellular and molecular mechanisms responsible for endothelial cell invasion represent a major focus of angiogenesis research throughout the last decade.

One of the earliest studies of the cellular and molecular mechanisms of angiogenesis addressed the degradation of major basement membrane structural components (i.e., collagen types IV and V) by migrating endothelial cells (18). In an in vitro assay, endothelial cells responded to a chemoattractant located on the opposite side of a filter containing collagen substrate. The investigators isolated metalloproteinases that degrade type IV or V collagens from membrane extracts of the migrating endothelial cells, demonstrating that endothelial cells express cell membrane-associated proteases specific for basement membrane collagens.

Studies on the effects of corticosteroids on angiogenesis have raised important issues regarding the role of proteolysis. Both cortisone and hydrocortisone, when administered with heparin or a heparin fragment, inhibited neovascularization in the chick embryo chorioallantoic membrane, the rabbit cornea, and selected mouse tumors (19). By contrast, treatment with heparin alone promoted tumor angiogenesis, while independent treatment with cortisone elicited little or no effect on angiogenesis. Furthermore, the angiostatic action of the administered corticosteroids was found to be independent of their glucocorticoid and mineralocorticoid activities (20,21). Steroids whose only observed function is the inhibition of neovascularization include the dihydro- and tetrahydrosteroid metabolites of cortisone, which may act physiologically to maintain angiostasis (19). Although the mechanism of corticosteroid-induced inhibition of neovascularization is unknown, it has been observed that corticosteroid and heparin co-administration induces basement membrane dissolution along capillaries that regress in response to such treatment (22). This seemingly paradoxical finding that basement membrane dissolution may inhibit rather than promote angiogenesis suggests a complex role for proteolysis in angiogenesis. While specific basement membrane proteolysis by endothelial cells invading the subendothelial matrix is integral to the expansion of a vascular network, it is likely that more generalized dissolution of the basement membrane and extracellular matrix (as elicited, for example, by corticosteroid and heparin co-administration) effectively removes the substrate within which endothelial cells are able to migrate and proliferate, and thus inhibits angiogenesis.

Because endothelial cells are not usually invasive, the elaboration of proteases involved in basement membrane degradation is believed to be tightly regulated (23). Numerous studies have sought to identify factors capable of inducing endothelial invasion in vitro. An important early investigation used bovine microvascular endothelial cells cultured on a collagen gel (24). Treatment of the endothelial cells with the tumor promoter phorbol myristate acetate (PMA), which dramatically increases capillary endothelial cell production of collagenase and plasminogen activator, induced invasive activity and subsequent capillary-like tube formation. This effect was prevented by treatment with the metalloproteinase inhibitor 1,10phenanthroline, a finding which supports the notion that endothelial cell invasiveness is a metalloproteinasedependent process. It is possible that PMA mimics the normal effects of angiogenic factors in vivo.

Pepper et al. modeled angiogenesis in vitro by wounding a confluent monolayer of bovine microvascular endothelial cells grown on gelatin substrate and then overlaying it with a casein-agar mixture containing plasminogen (25). Cell migration from the wound edge coincided with increased urokinase-type plasminogen activator (uPA) cell-surface expression, which returned to normal levels upon cessation of movement. 
Plasminogen activators produced by endothelial cells convert plasminogen to plasmin, a serine protease capable of directly degrading extracellular matrix components such as laminin and fibronectin. Plasmin may also act as an activator of the zymogen forms of secreted collagen-specific proteases, including type IV collagenase, which is essential for basement membrane degradation. This study thus substantiates previous findings implicating plasminogen activator in invasive proteolysis (24). To what extent this wounded monolayer model relates to angiogenesis during embryonic development and organogenesis, however, remains in question.

A crucial investigation involving bovine capillary endothelial cell growth on the human amnion basement membrane provided further insight into the role of basement membrane invasion during angiogenesis (26). Treatment of either the basement membrane or the underlying stromal aspect of the amnion with basic fibroblast growth factor (bFGF, also designated FGF-2) induced endothelial cell invasion in a dose-dependent manner. Several substances inhibited the invasion process, including transforming growth factor-beta (TGFß), inhibitors of plasminogen activators, anti-tissue plasminogen activator (tPA) antibody, a metalloproteinase inhibitor, and antibodies to type IV and interstitial collagenases. The results of this study are significant in their suggestion that angiogenesis involves degradation of both the perivascular basement membrane and the stroma of the tissue to be vascularized. This indicates that these processes are mediated by both direct and plasminogen-mediated protease activity.

Another significant discovery regarding the control of basement membrane proteolysis during angiogenesis arose from an in vitro model in which bovine microvascular endothelial cells cultured on fibrin gels were treated with various angiogenic factors (27). Prompted by a seemingly counterintuitive earlier report that the angiogenesis inducer bFGF stimulates the production of plasminogen activator inhibitor-1 (PAI-1) by endothelial cells (28), Pepper et al. showed that the angiogenic agents TGF- 31 and PMA also induce PAI-1 (27). Interestingly, they observed that TGF- $\beta 1$ inhibited lumen formation in tube-like structures that had been induced by bFGF. Considerable progress toward elucidating the mechanism for this phenomenon was made by measuring the ratio of uPA:PAI-1 mRNA levels as an indicator of proteolytic balance. In response to bFGF, the balance is shifted toward enhanced proteolysis; that is, more UPA is induced than PAI-1. By contrast, TGF- $\beta 1$ tilts the balance toward inhibition of proteolysis through greater induction of PAI-1 than uPA. As shown in Table 1, TGF- $\beta$ also modulates angiogenesis through inhibition of endothelial cell motility and proliferation (29). The cytostatic effect of TGF- $\beta 1$ is likely attributable to the induction of cell cycle arrest via the inhibition of a number of steps involved in cyclin-dependent kinase gene ( $c d k)$ activation, thus preventing the phosphorylation of the Rb gene in late G1 phase (30). These observed inhibitory effects of TGF- $\beta$ on proteolysis and endothelial cell motility and proliferation are further complicated by the finding that, in vivo, TGF- $\beta 1$ can elicit monocyte-mediated induction of angiogenesis (31). TGF- $\beta 1$ may thus have two opposing activities in vivo: direct-acting inhibition and monocyte-mediated induction of angiogenesis.

Matrix metalloproteinase enzymes and their specific inhibitors from another enzyme-inhibitor system modulating basement membrane degradation during angiogenesis. The tissue inhibitors of metalloproteinases (TIMP-1 and TIMP-2) bind several members of the metalloproteinase family, including active interstitial collagenase and latent and active type IV collagenases, $(23,32)$. The suggestion that TIMPs inhibit the protease activity of active metalloproteinases and/or prevent stromelysin-mediated activation of latent metalloproteinases provided the conceptual basis for further in vitro investigations of angiogenesis using these inhibitors (32). TIMP-1 and general inhibitors of both serine- and metalloproteinases have demonstrated antiangiogenic activity in the in vitro human amniotic membrane model (25). Furthermore, the balance between type IV collagenases and TIMPs was recognized as a key determinant of basement membrane proteolysis during endothelial tube formation on a collagen substrate (33).

Research on laminin, a major basement membrane glycoprotein, has revealed an additional layer of complexity in the modulation of basement membrane proteolysis during angiogenesis. In vitro studies demonstrate that laminin, previously known to promote cell migration by haptotaxis, contains both an 
angiogenic and an antiangiogenic peptide unit (34). The angiogenic peptide increases collagenase IV activity and plasminogen activator activation, and is located on a chain of the laminin molecule whose expression varies during development (8). Therefore, it is possible that laminin plays a role in the induction of angiogenesis during embryonic development and organogenesis, while inhibiting the process in more mature tissues. Furthermore, future research may reveal that, in some pathological forms of angiogenesis, mutations in the genetic regulator(s) of the embryonically-expressed laminin chain gene promote the expression of this protein in mature tissues. A strong analogy is evident between this hypothetical mechanism underlying certain angiogenic phenomena and the concept of proto-oncogene activation during tumorigenesis.

\section{ENDOTHELIAL CELL MOTILITY AND PROLIFERATION}

A pervasive feature among a number of angiogenic agents is their ability to stimulate endothelial cells at three levels: proteolysis, motility, and proliferation (23). As discussed above, proteolysis of matrix components by endothelial cells is required for egress from the parent vessel, as well as for capillary sprout penetration into, and lateral expansion within, the extracellular matrix. Motility is involved in both endothelial cell chemotaxis toward angiogenic stimuli and endothelial cell alignment to form a capillary sprout. Lastly, endothelial cell proliferation is essential in order to populate the expanding neovascular network.

Tumors grown in laboratory animals yielded the first isolated angiogenic factors $(4,35)$. The advent of endothelial cell cultures provided a further impetus for the isolation of angiogenic factors, particularly endothelial cell growth factors. With the discovery that many of these factors bind strongly to heparin, the application of heparin affinity chromatography facilitated the purification of endothelial cell growth factors from a variety of non-neoplastic tissues, including the retina, hypothalamus, brain, and cartilage (4).

Angiogenic factors can be classified according to a variety of characteristics, including the effect on endothelial cells, the mechanism of action, and the molecular form. Many of the angiogenic agents that induce endothelial cell motility are believed to be chemotactic factors, while agents that induce proliferation generally belong to the growth factor family (2). Among the substances acting principally to induce either endothelial cell motility or proliferation, many target endothelial cells directly, while others are thought to act indirectly, their effects mediated by other host cells. Furthermore, angiogenic agents can exist either in secreted form or, in the case of such molecules as epidermal growth factor (EGF) and transforming growth factor-alpha (TGF-a), as membrane-bound glycoproteins (13). Bound angiogenic molecules are cleaved from the endothelial cell membrane to yield a soluble mitogen or chemotactic factor. Table 1 shows the biological activities of the major angiogenic factors associated with endothelial cell motility and proliferation.

Two of the earliest factors isolated were endothelial cell growth factor (ECGF) from the hypothalamus and fibroblast growth factor (FGF) from the brain; these molecules are now understood to be structurally related (2). ECGF, as originally described, is a precursor to what has become known as acidic fibroblast growth factor (aFGF, or FGF-1), while the brain-derived FGF has since been redesignated as basic FGF (bFGF, or FGF-2).

FGFs are highly potent angiogenic factors, and are commonly used to initiate blood vessel development in in vitro models in which the effects of subsequently administered modulators of angiogenesis are to be studied. FGF-1 and -2 are bound to low-affinity heparan sulfate sites in the extracellular matrix, and must be released by heparitinase or matrix-degrading proteases, such as plasminogen activator, in order to bind to high-affinity cellular receptors and thereby exert their biological effects $(2,36,37)$. As shown in Table 1, FGFs function dually as direct-acting endothelial cell mitogens and chemotactic factors (2).

Vascular endothelial growth factor (VEGF, also referred to as vascular permeability factor, or VPF) is another heparin-binding endothelial cell growth factor known to possess angiogenic activity in vivo $(38,39)$. A 
structural homolog of platelet-derived growth factor (PDGF), VEGF was first isolated as a tumor-derived vascular permeability factor, and exists in one of four different molecular species arising by alternative mRNA splicing (38). Two of these are soluble proteins, whereas the other two are bound to cell-surface or basement membrane proteoglycans containing heparan. As indicated in Table 1, VEGF acts directly on endothelial cells to induce cell proliferation (38-41) and migration $(5,42)$. The precise mechanism, although unknown, is thought to involve tyrosine kinase receptor-mediated activation of phospholipase $\mathrm{C}$, leading to a transient rise in intracellular calcium concentration (43). Investigations have shown that VEGF may be induced by hypoxia to promote angiogenesis in ischemic tissues (40). It is possible that the low oxygen tension in embryonic tissues and developing organs likewise induces VEGF to promote neovascularization of these regions.

Several angiogenic processes are associated with the menstrual cycle. The extensive neovascularization that occurs in the periphery of developing ovarian follicles, and the microvascular expansion into neighboring lutein cells that accompanies the development of corpora lutea, may both depend on VEGF (44). The expression of VEGF mRNA by perivascular cells has been documented in female mice during the neovascularization of ovarian follicles and the corpus luteum, and during the expansion of the endometrial vasculature (45). Moreover, VEGF can be expressed in numerous steroidogenic and/or steroid-responsive cell types, including theca, lutein, granulosa, endometrial stroma, decidua, and adrenal cortical cells; in some cases, VEGF expression is restricted to a particular phase of the ovarian cycle (46).

In addition to the mitogenic role of VEGF in angiogenesis, a complementary angiogenic function of this molecule has recently been proposed that implicates VEGF in the induction of microvascular hyperpermeability. Endothelial permeability results in extravasation of plasma proteins into the extracellular space. This leads to the deposition of a fibrin gel that serves as a provisional matrix fostering the ingrowth of new blood vessels. In various physiological and pathological processes, microvascular hyperpermeability and plasma-derived matrix deposition correlate with the onset of angiogenesis (46). It is therefore possible that microvascular hyperpermeability is a crucial feature of angiogenesis, and that VEGF, as a unique inducer of vessel permeability, may be a ubiquitous player in the neovascularization process. In this regard, VEGF is thought to interact with other angiogenic factors, as evidenced by its regulation tissue factor (TF) expression and its role in mediating, at least partially, the effects of FGF-2, TGF-*, tumor necrosis factor, histamine, and other agents $(43,46)$.

Platelet-derived growth factor (PDGF) may also act as an endogenous modulator of angiogenesis. The expression of PDGF mRNA by microvascular endothelial cells is increased by pro-inflammatory factors such as TGF- $\beta$ and thrombin, and is decreased by agents that elevate cAMP (47). In conventional two-dimensional culture, endothelial cells proliferate and express alpha and beta chains of the PDGF receptor. However, in three-dimensional culture, capillary-like tube formation and the corresponding induction of a nonproliferative, differentiated endothelial cell phenotype is associated with downregulation of PDGF receptors (48). This is reflected in Table 1, which shows that PDGF induces endothelial cell proliferation. As evidenced by the differential expression of PDGF receptors in the progression of endothelial cell proliferation to sprout development and subsequent tube formation, the plasticity of the endothelial cell phenotype is an important feature of the angiogenic process.

Platelet-activating factor (PAF), a phospholipid mediator of inflammation, is produced by stimulated monocytes/macrophages, neutrophils, basophils, and platelets, and is also elaborated by cultured endothelial cells after stimulation by a variety inflammatory mediators, such as histamine and TNF-* (49). Therefore, the angiogenic response to histamine and TNF-* may, at least in part, be mediated by PAF. Additionally, an autocrine mechanism for PAF may be suggested by the evidence that endothelial cells both produce PAF and express PAF-specific receptors. At physiological concentrations, PAF induces endothelial cell migration, but not proliferation (50). PAF exhibits a range of other biological effects, including the enhancement of vascular permeability, which may have implications in angiogenesis similar to those discussed above for VEGF. 
The potential role of tissue factor (TF, also known as thromboplastin) in angiogenesis was recently studied by transfecting sarcoma cells with sense and antisense constructs of the TF gene (51). Tumor cells overexpressing TF established larger and more vascular tumors than both antisense transfectants (which underexpressed TF) and untransfected tumor cell controls. The degree of TF expression by tumor cells was positively correlated with the endothelial cell mitogenic response. Furthermore, tumor cell TF expression varied directly with the transcription of VEGF and inversely with that of thrombospondin-2 (TSP-2). TF, the principal molecule of the extrinsic coagulation pathway, is expressed by a variety of tumors, and is hypothesized to mediate the enhanced procoagulant activity common among cancer patients (52). However, the proposed induction of angiogenesis by TF, and its consequent significance in tumor growth, are most likely independent of its interaction in the coagulation cascade (51). The extent to which TF may promote angiogenesis in physiological and non-neoplastic pathological states is currently unknown.

Thrombospondin-1 (TSP-1) is a perivascular extracellular matrix glycoprotein secreted by platelets, endothelial cells, and a variety of other cells; it stabilizes platelet aggregates in thrombi and wounds $(53,54)$. Five unique members of the thrombospondin family have been identified to date; among them, only TSP-1 and -2 contain domains likely to influence angiogenesis (54). Loss of p53 in fibroblasts cultured from patients with Li-Fraumeni syndrome leads to reduced expression of TSP-1 and concomitant expression of the angiogenic phenotype (55). This is explained by the observation that, as shown in Table 1, TSP-1 inhibits in vitro endothelial cell migration and proliferation toward angiogenic agents (56). TSP-1 also inhibits neovascularization in vivo (54). In a recent study, however, human peripheral blood monocytes (which are recognized as proangiogenic by the rat cornea assay) were found to produce TSP-1, an angiogenic inhibitor (57). This paradoxical finding draws attention to the fact that macrophages produce both angiogenic and antiangiogenic agents, and to the likelihood that the net biological effect may reflect a balance in the modulators produced. Clearly, it is difficult to interpret angiogenic responses through the analysis of only a single angiogenic mediator.

As presented in Table 1, heparin produced by activated mast cells potentiates angiogenesis by stimulating endothelial cell locomotion (58). Additional evidence suggests that heparin induces endothelial cell proliferation and motility indirectly by increasing the binding of FGFs to their endothelial receptors, as well as by protecting FGFs from inactivation (2). Heparan sulfate proteoglycans are the major glycosaminoglycans on the surface of endothelial cells and in the subendothelial basement membrane (59). Through their strong affinity for many endothelial cell growth factors, heparin-like molecules on the endothelial cell surface may assist in the angiogenic process by serving to concentrate the growth factors in the immediate vicinity of the vascular endothelium (4).

Heparin is prototypical of those angiogenic factors that act by indirect pathways and thus, despite their in vivo angiogenic activity, may not demonstrate an effect on vascular endothelial cells in vitro. Several indirect pathways are possible. The factors may, for example, mobilize monocytes and activate them to secrete endothelial cell mitogens and/or chemotactic factors. Alternatively, indirect angiogenic factors may cause the release of matrix-bound or intracellularly-stored, direct-acting angiogenic factors.

Nitric oxide (NO), also known as endothelial-derived relaxation factor (EDRF), has recently been added to the growing list of angiogenic modulators. In both in vitro endothelial cell cultures and in vivo in the rat cornea, capillary endothelial cell migration and proliferation was induced in a dose-dependent manner by exogenous NO generators and by endogenous NO production elicited by substance P (60). As is true for PAF, endothelial cells both produce and respond to NO.

A large variety of other angiogenic modulators are currently undergoing early investigation. For example, TIMP-1 and -2 have been shown to inhibit tumor invasion and angiogenesis in vitro by inhibiting metalloproteinase activity and thereby blocking tumor and endothelial cell motility (61). Quite interestingly, it has been shown recently that TIMP-2 can inhibit bFGF-induced endothelial cell proliferation by an as yet 
undefined mechanism that is independent of metalloproteinase inhibition (32). Similarly, somatostatin analogs such as octreotide acetate have been shown to inhibit angiogenesis in vitro, and although the precise mechanism is unclear, it is thought to involve direct endothelial cell activation and to be $\mathrm{G}$ protein-, calciumand cAMP-dependent $(62,63)$. In addition, an internal fragment of prolactin (PRL) potently inhibits angiogenesis modeled in vitro by bFGF-induced proliferation of cultured endothelial cells (64). Native PRL, however, appears to have no effect, raising the possibility that some modulators of neovascularization arise by specific proteolysis of circulating, angiogenically-inactive molecules. Recombinant ribonuclease (RNase) inhibitor has also been implicated as a modulator of angiogenesis, inhibiting the angiogenic response to bFGF in the mouse cornea (65). Subcutaneous implantation of RNase inhibitor-releasing disks beneath intradermally-inoculated mammary tumor cells showed significant inhibition of tumor growth. Furthermore, the novel angiogenesis inhibitor angiostatin, which is homologous to an internal segment of plasminogen, appears in the serum in the presence of a primary tumor and markedly suppresses angiogenesis in distant metastases (66). Lastly, numerous macrophage secretory products, including several mentioned previously, have been shown to mediate angiogenesis (67).

Given the great diversity of angiogenic factors and their pluralistic modes of action and biological activities, physiological mechanisms to prevent rampant capillary neoformation must be equally complex. Indeed, under most normal conditions, capillary endothelial cells remain quiescent. The regulatory mechanisms responsible for this quiescence plausibly involve both intracellular controls, such as growth factor sequestration, and extracellular controls, including inhibitors such as TGF-ß3.

\section{CLINICAL APPLICATIONS}

A flurry of activity in the field of angiogenesis research is currently underway as its expanding clinical contexts are continuously being discovered. In particular, a potential role of angiogenic modulation has been suggested in diabetic retinopathy, rheumatoid arthritis, cardiovascular disease, certain vasculopathies, tissue grafting and organ transplantation, and in various solid tumors, such as Kaposi's sarcoma. The visionthreatening vascular proliferation in diabetic retinopathy has been postulated to stem from the stimulation of choroidal endothelial cells by VEGF and FGF-2 elaborated by retinal pigment epithelial cells (68). By contrast, the release of angiogenic inhibitors by retinal pigment epithelial cells has been proposed as the physiological mechanism underlying avascularity in the retina (69).

The administration of the AGM-1470 (TNP-470) derivative of fumagillin, an angiogenesis inhibitor obtained from Aspergillus fumigatus, has proven effective in preventing neovascularization of joint synovia in rat models of rheumatoid arthritis (70). Synovial fluid VEGF levels have been shown by immunoassay to be significantly higher in rheumatoid arthritis patients than in those with other arthritides (5). Immunohistologic studies have demonstrated p55 and p70 IL-2 receptors in the vasculature of rheumatoid joints (71), suggesting possible molecular targets for future therapies.

In another application of angiogenesis research, direct gene transfer of an expression vector for FGF-1 into porcine arteries shows promise for the neovascularization and proposed resolution of early atherosclerotic lesions (72). Other studies have demonstrated that angiogenic growth factor-laden fibrin glue, implanted between the aorta and left ventricular myocardium, induced site-directed neovascular development (73). Related studies have shown enhanced de novo epicardial small vessel growth in rabbits receiving intrapericardial infusions of FGF-2, especially in those animals having left ventricular hypertrophy (74). This provides exciting prospects for intervention in cardiac diseases in which myocardial oxygen supply is compromised and/or demand is increased. It is quite conceivable that the induction of new collateral vessel formation may delay or halt the progression of coronary artery disease to myocardial infarction, as well as the advancement of ventricular hypertrophy to heart failure.

With respect to vasculopathies, clinical trials are being performed in the treatment of high-risk hemangiomas 
of infancy through long-term daily administration of inteferon-*-2*, which has anti-angiogenic properties (75). In addition, in the chronic inflammatory condition of systemic vasculitis, increased levels of angiogenic haptoglobin in patient sera have been implicated in the hyperproliferation of vessels, possibly in response to ischemia of involved tissues (76).

Angiogenic modulation has also been applied to peptic ulcer disease. As a peptic ulcer is essentially a form of wound, one would expect its healing to depend upon microvascular ingrowth. Indeed, the induction of angiogenesis in duodenal ulcer beds via oral administration of acid-resistant FGF-2 in rats has successfully accelerated healing of the ulcers, and is currently undergoing Phase I clinical trials $(77,78)$.

Recent investigation of pancreatic islet iso- and xenografting in the hamster have demonstrated that endothelial cells of host muscle tissue origin are responsible for revascularization of the grafts (79). Since graft endothelial cell-dependent antigen recognition by host immune cells, a host-versus-graft reaction, is thought to play an important role in most graft rejections, this result prompts the hypothesis that graft survival could be enhanced if the selective inhibition of neovascularization in graft tissue and promotion of angiogenesis in host tissue is achieved.

Perhaps the most important clinical applications to emerge from angiogenic research lie in cancer therapy. Phase I trials using carboxy-amino-triazole (CAT), an anti-angiogenic agent, are in progress for the treatment of cancer patients with various tumor types (80). A fumagillin analog that has demonstrated tumoristatic activity against Lewis lung carcinoma and B12 melanoma is also presently undergoing clinical trials. IFN-*, which suppresses angiogenesis in vivo, has been approved by the U.S. Food and Drug Administration for the treatment of Kaposi's sarcoma in AIDS patients (81). Cultured Kaposi's sarcoma cells exhibit PAF production and release, and possess high-affinity, membrane-associated PAF receptors (82), providing molecular targets that could be exploited in alternative therapy of this neoplasm. In addition, antisense oligonucleotides directed against FGF-2 mRNA have significantly inhibited proliferation of Kaposi's sarcoma cells derived from AIDS patients and suppressed the demonstrated angiogenic activity of these cells (83).

Furthermore, angiogenesis is also being used in tumor grading, the density of vascularization positively correlating with the risk of tumor progression (84). The use of microvessel quantitation, as well as immunohistochemical staining for endothelial cell markers, in the pathological evaluation of neoplasms promises to become more prevalent in the future.

\section{CONCLUSION}

Advances in the elucidation of angiogenesis in recent years stem primarily from the investigation of three critical features of the neovascularization process, namely endothelial cell proliferation, motility, and basement membrane proteolysis. Although considerable progress has been made with respect to the characterization of angiogenic factors that mediate these processes, much remains to be discovered concerning angiogenic regulatory mechanisms.

In this regard, it appears that the primary difference between tumor angiogenesis and normal neovascularization lies not in the elaboration of distinct endothelial cell growth factors, but rather in the modulation of their expression and activity. Whereas tumors most often express angiogenic agents continuously, many normal tissues seem to contain endothelial factors that are only expressed under tight regulation. The diverse activities and modes of action of the numerous angiogenic modulators greatly complicate our understanding of the regulatory mechanisms involved in neovascularization.

In the final analysis, when dealing with such a complex phenomenon as angiogenesis, the sharp distinction between angiogenic factors direct versus indirect, protease-activating versus chemotactic or mitogenic, and even inducing versus inhibiting is quite likely an oversimplification. Some angiogenic factors exert both 
direct and indirect effects, while others function dually as mitogens and activators of proteolysis. Still other angiogenic factors act as inducers in some contexts and inhibitors in others. Further research into angiogenesis may show that, like tumor cell release of VEGF, the expression of angiogenic factors responds to signals from the cellular microenvironment. Future discoveries may indeed reveal that the temporally coordinated activities of a number of key angiogenic regulators are responsible for the predominance of neovascularization during embryogenesis and various physiological and pathological processes, including tumor metastasis.

\section{REFERENCES}

1. Benjamini E, Leskowitz S. Immunology: a short course, 2nd ed. New York: Wiley-Liss, Inc.; 1991

2. Folkman J, Klagsbrun M. Angiogenic factors. Science 235: 442-447, 1991.

3. Folkman J. What is the evidence that tumors are angiogenesis dependent? Journal of the National Cancer Institute 82: 4-6; 1990.

4. Folkman J. Toward an understanding of angiogenesis: search and discovery. Perspectives in Biology and Medicine 29(1): 10-36; 1985.

5. Koch AE, et al. Vascular endothelial growth factor. A cytokine modulating endothelial function in rheumatoid arthritis. Journal of Immunology 152(8): 4149-4156; 1994.

6. Murphy RP. Management of diabetic retinopathy. American Family Physician 51(4): 785-96; 1995.

7. Ausprunk DH, Folkman J. Migration and proliferation of endothelial cells in preformed and newly formed blood vessels during tumor angiogenesis. Microvascular Research 14: 53-65; 1977.

8. Kleinman HK, et al. The laminins: a family of basement membrane glycoproteins important in cell differentiation and tumor metastases. Vitamins and Hormones 47: 161-184; 1993.

9. Yanase T, et al. Inhibitory effect of angiogenesis inhibitor TNP-470 on tumor growth and metastasis of human cell lines in vitro and in vivo. Cancer Research 53: 2566-2570; 1993.

10. Gimbrone MA Jr., Cotrans RS, Folkman J. Human vascular endothelial cells in culture: growth and DNA synthesis. Journal of Cell Biology 60: 673-680; 1974.

11. Ausprunk DH, Knighton DR, Folkman J. Vascularization of normal and neoplastic tissues grafted into the chick chorioallantois. American Journal of Pathology 79: 597-618; 1975.

12. Passaniti A, et al. A simple, quantitative method for assessing angiogenesis and antiangiogenic agents using reconsitituted basement membrane, heparin, and fibroblast growth factor. Laboratory Investigation 67: 519-28; 1992.

13. Gleave ME, et al. Epidermal growth factor receptor-mediated autocrine and paracrine stimulation of human transitional cell carcinoma. Cancer Research 53: 5300-5307; 1993.

14. Folkman J, et al. Induction of angiogenesis during the transition from hyperplasia to neoplasia. Nature 339: 58-61, 1989.

15. Browder LW, Erickson CA, Jeffery WR. Developmental Biology, 3rd ed. Philadelphia, PA: Saunders College Publishing; 1991. 
16. Schor SL. Cell proliferation and migration on collagen substrate in vitro. Journal of Cell Science 41: 159$175 ; 1980$.

17. Delvos U, et al. Interaction of vascular wall cells with collagen gels. Laboratory Investigations 46: 61-72; 1982.

18. Kalebic T, et al. Basement membrane collagen: degradation by migrating endothelial cells. Science 221 : 281-283; 1982.

19. Folkman J, et al. Angiogenesis inhibition and tumor regression caused by heparin or a heparin fragment in the presence of cortisone. Science 221: 719; 1983.

20. Crum R, Szabo S, Folkman J. A new class of steroids inhibits angiogenesis in the presence of heparin or a heparin fragment. Science 230: 1375; 1985.

21. Crum R, Folkman J. Anti-angiogenesis by steroids without glucocorticoid or mineralocorticoid activity in the presence of heparin. Journal of Cell Biology 99: 158a; 1984.

22. Ingber DE, Madri JA, Folkman J. Anti-angiogenesis by angiostatic steroids in the presence of heparin: induction of basement membrane dissolution. Journal of Cell Biology 101: 105a; 1985.

23. Liotta LA, Steeg PS, Stetler-Stevenson WG. Cancer metastasis and angiogenesis: an imbalance of positive and negative regulation. Cell 64: 327-336; 1991.

24. Montesano R, Orci L. Tumor-promoting phorbol esters induce angiogenesis in vitro. Cell 42: 469-477; 1985.

25. Pepper MS, et al. Urokinase-type plasminogen activator is induced in migrating capillary endothelial cells. Journal of Cell Biology 105: 2535-2541; 1987.

26. Mignatti $\mathrm{P}$, et al. In vitro angiogenesis on the human amniotic membrane: requirement for basic fibroblast growth factor-induced proteinases. Journal of Cell Biology 108: 671-682; 1989.

27. Pepper MS, et al. Transforming growth factor-beta 1 modulates basic fibroblast growth factor-induced proteolytic and angiogenic properties of endothelial cells in vitro. Journal of Cell Biology 111: 743-755; 1990.

28. Saksela O, Moscatelli D, Rifkin DB. The opposing effects of basic fibroblast growth factor and transforming growth factor beta on the regulation of plasminogen activator activity in capillary endothelial cells. Journal of Cell Biology 105: 957-963; 1987.

29. Muller $\mathrm{G}$, et al. Inhibitory action of transforming growth factor beta on endothelial cells. Proceedings of the National Academy of Sciences USA 84: 5600-5604; 1987.

30. Lin HY, Moustakas A. Cellular and Molecular Biology 40(3): 337-349; 1994.

31. Weisman DM, et al. Transforming growth factor-beta is chemotactic for human monocytes and induces their expression of angiogenic activity. Biochemical and Biophysical Research Communications 157: 793$800 ; 1988$.

32. Murphy AN, Unsworth EJ, Stetler-Stevenson WG. Tissue inhibitor of metalloproteinase-2 inhibits bFGFinduced human microvascular endothelial cell proliferation. Journal of Cellular Physiology 157(2): 351-358; 
1993.

33. Schnapper HW, et al. Type IV collagenases and TIMPs modulate endothelial cell morphogenesis in vitro. Journal of Cellular Physiology 156: 235-246; 1993.

34. Grant DS, et al. Two different laminin domains mediate the differentiation of human endothelial cells into capillary-like structures in vitro. Cell 58: 933-943; 1989.

35. Azizkhan J, et al. Stimulation of increased capillary endothelial cell motility by chondrosarcoma cellderived factors. Cancer Research 43(7): 3281-3286; 1983.

36. Bashkin P, et al. Basic fibroblast growth factor binds to subendothelial extracellular matrix and is released by heparitinase and heparin-like molecules. Biochemistry 28: 1737-1743; 1989.

37. Friesel RE, Maciag T. Molecular mechanisms of angiogenesis: fibroblast growth factor signal transduction. FASEB 9(10): 919-925; 1995.

38. Kim KJ, et al. Inhibition of vascular endothelial growth factor-induced angiogenesis suppresses tumour growth in vivo. Nature 362: 841-844; 1993.

39. Plate KH, et al. Vascular endothelial growth factor is a potential tumor angiogenesis factor in human gliomas in vivo. Nature 359: 845-848; 1992.

40. Shweiki D, et al. Vascular endothelial growth factor induced by hypoxia may mediate hypoxia-initiated angiogenesis. Nature 359: 843-845; 1992.

41. Connolly DT, et al. Tumor vascular permeability factor stimulates endothelial cell growth and angiogenesis. Journal of Clinical Investigation 84: 1470-1478; 1989.

42. Favard C, et al. Purification and biological properties of vasculotropin, a new angiogenic cytokine. Biology of the Cell 73(1): 1-6; 1991.

43. Connolly DT. Vascular permeability factor: a unique regulator of blood vessel function. Journal of Cell Biochemistry 47(3): 219-23; 1991.

44. Findlay JK. Angiogenesis in reproductive tissues. Journal of Endocrinology 111: 357-366; 1986.

45. Shweiki D, et al. Patterns of expression of vascular endothelial growth factor (VEGF) and VEGF receptors in mice suggest a role in hormonally regulated angiogenesis. Journal of Clinical Investigation 91(5): 2235-2243; 1993.

46. Dvorak HF, et al. Vacular permeability factor/vascular endothelial growth factor, microvascular hyperpermeability, and angiogenesis. American Journal of Pathology 146(5): 1029-1039; 1995.

47. Kavanaugh WM, et al. Transcriptional regulation of the A and B chain genes of platelet-derived growth factor in microvascular endothelial cells. Journal of Biological Chemistry 263: 8470-8472; 1988.

48. Marx M, Perlmutter RA, Madri JA. Modulation of platelet-derived growth factor receptor expression in microvascular endothelial cells during in vitro angiogenesis. Journal of Clinical Investigation 93(1): 131-139; 1994.

49. Camussi G, et al. The release of platelet-activating factor from human endothelial cells in culture. Journal 
of Immunology 131: 2397-2403; 1983.

50. Camussi G, et al. Platelet-activating factor directly stimulates in vitro migration of endothelial cells and promotes in vivo angiogenesis by a heparin-dependent mechanism. Journal of Immunology 154(12): 6492$6501 ; 1995$.

51. Zhang Y, et al. Tissue factor controls the balance of angiogenic and antiangiogenic properties of tumor cells in mice. Journal of Clinical Investigation 94(3): 1320-1327; 1994.

52. Murray, JC. Coagulation and cancer. British Journal of Cancer 64: 422-424; 1991.

53. Tolsma $S$, et al. Peptides derived from two separate domains of the matrix protein thrombospondin-1 have anti-angiogenic activity. Journal of Cell Biology 122: 497-511; 1993.

54. Good DJ, et al. A tumor suppressor-dependent inhibitor of angiogenesis is immunologically and functionally indistinguishable from a fragment of thrombospondin. Proceedings of the National Academy of Sciences USA 87: 6624-6628; 1990.

55. Dameron KM, et al. Control of angiogenesis in fibroblasts by p53 regulation of thrombospondin- 1 . Science 265: 1582-1584; 1994.

56. Taraboletti G, et al. Platelet thrombospondin modulates endothelial cell adhesion, motility, and growth: a potential angiogenesis regulatory factor. Journal of Cell Biology 111: 765-772; 1990.

57. DiPietro LA, Polverini PJ. Angiogenic macrophages produce the angiogenic inhibitor thrombospondin 1. American Journal of Pathology 143(3): 678-684; 1993.

58. Azizkhan RG, et al. Mast cell heparin stimulates migration of capillary endothelial cells in vitro. Journal of Experimental Medicine 152(4): 931-944; 1980.

59. Buonassisi V, Root M. Enzymatic degradation of heparin-related mucopolysaccharides from the surface of endothelial cell cultures. Biochimica et Biophysica Acta 385(1): 1-10; 1975.

60. Ziche M, et al. Nitric oxide mediates angiogenesis in vivo and endothelial cell growth and migration in vitro promoted by substance P. Journal of Clinical Investigation 94(5): 2036-2044; 1994.

61. Ray JM, Stetler-Stevenson WG. The role of matrix metalloproteinases and their inhibitors in tumor invasion, metastasis and angiogenesis. European Respiratory Journal 7: 2062-2072; 1994.

62. Wolfering EA, et al. Somatostatin analogues inhibit angiogenesis in the chick chorioallantoic membrane. Journal of Surgical Research 50: 245-251; 1991.

63. Patel PC, et al. Postreceptor signal transduction mechanisms involved in octreotide-induced inhibition of angiogenesis. Surgery 116(6): 1148-1152; 1994.

64. Clapp C, et al. The 16-kilodalton N-terminal fragment of human prolactin is a potent inhibitor of angiogenesis. Endocrinology 133: 1292-1299; 1993.

65. Polakowski IJ, et al. A ribonuclease inhibitor expresses anti-angiogenic properties and leads to reduced tumor growth in mice. American Journal of Pathology 143(2): 507-517; 1993.

66. Oreilly MS, et al. Angiostatin: a novel angiogenesis inhibitor that mediates the suppression of metastases 
by a Lewis lung carcinoma. Cell 79: 315-328; 1994.

67. Sunderköpter C, et al. Macrophage and angiogenesis. Journal of Leukocyte Biology 55: 410-422; 1994.

68. Sakamoto T, et al. Vessel formation by choroidal endothelial cells in vitro is modulated by retinal pigment epithelial cells. Archives of Ophthalmology 113(4): 512-520; 1995.

69. Glaser B, et al. Retinal pigment epithelial cells release inhibitors of neovascularization. Ophthalmology 94: 780; 1983 .

70. Folkman J. Angiogenesis in cancer, vascular, rheumatoid and other disease. Nature Medicine 1(1): 27-31; 1995.

71. Cozzolino F, et al. Interferon-alpha and interleukin 2 synergistically enhance basic fibroblast growth factor synthesis and induce release, promoting endothelial cell growth. Journal of Clinical Investigation 91(6): 2504-2512; 1993.

72. Nabel EG, et al. Recombinant fibroblast growth factor-1 promotes intimal hyperplasia and angiogenesis in arteries in vivo. Nature 362: 844-846; 1993.

73. Fasol R, et al. Experimental use of a modified fibrin glue to induce site-directed angiogenesis from the aorta to the heart. Journal of Thoracic and Cardiovascular Surgery 107(6): 1432-1439; 1994.

74. Landau C, Jacobs AK, Haudenschild, CC. Intrapericardial basic fibroblast growth factor induces myocardial angiogenesis in a rabbit model of chronic ischemia. American Heart Journal 129(5): 924-931; 1995.

75. Ezekowitz RAB, Mulliken JB, Folkman J. Interferon alpha-2a therapy for life-threatening hemangiomas of infancy. New England Journal of Medicine 326: 1456-1463; 1992 (and corrections New England Journal of Medicine 330: 300; 1994).

76. Cid MC, et al. Identification of haptoglobin as an angiogenic factor in sera from patients with systemic vasculitis. Journal of Clinical Investigation 91(3): 977-985; 1993.

77. Hull MA, Cullen DJE, Hawkey CJ. Basic fibroblast growth factor in gastric ulceration: mucosal levels and therapeutic potential. Gastroenterology 106: A97; 1994.

78. Wolfe MM, et al. Safety and efficacy of an angiogenic peptide, basic fibroblast growth factor (bFGF) in the treatment of gastroduodenal ulcers: a preliminary report. Gastroenterology 106: A212; 1994.

79. Vajkoczy P, et al. Histogenesis and ultrastructure of pancreatic islet graft microvasculature. Evidence for graft revascularization by endothelial cells of host origin. American Journal of Pathology 146(6): 1397-1405; 1995.

80. Kohn E, et al. Phase I trial of signal transduction inhibitor, CAI. Proceedings of the American Association of Cancer Research 35: 244; 1994.

81. Logan A. Angiogenesis. Lancet 341: 1467-1468; 1993.

82. Bussolino F, et al. Platelet activating factor produced in vitro by Kaposi's sarcoma cells induces and sustains in vivo angiogenesis. Journal of Clinical Investigation 96(2): 940-952; 1995. 
83. Ensoli B, et al. Block of AIDS-Kaposi's sarcoma (KS) cell growth, angiogenesis, and lesion formation in nude mice by antisense oligonucleotide targeting basic fibroblast growth factor. A novel strategy for the therapy of KS. Journal of Clinical Investigation 94(5): 1736-1746; 1994.

84. Hollingsworth HC, et al. Tumor angiogenesis in advanced stage ovarian carcinoma. American Journal of Pathology 147(1): 33-41; 1995.

\section{BIOGRAPHY}

Neil A. Goldenberg graduated cum laude from Williams College (Williamstown, Massachusetts) in 1993 with a bachelor's degree in English, and is currently in the Class of 1998 at the McGill University Faculty of Medicine (Montreal, Quebec). The author served on the editorial staff of Genetic Engineering News (New York, New York) in the summer of 1990, and has research experience in tumor biology and immunology, including the development of monoclonal antibody conjugates with cytotoxic drugs at the Garden State Cancer Center (Newark, New Jersey), and in vitro studies of antileukemic chemotherapeutics at UMD-New Jersey Medical School. In 1992 and 1993, he was awarded summer research fellowships at the National Institute of Health to assist in the development of recombinant viral vaccines in cancer therapy.

Copyright (C) 1995 by MJM 